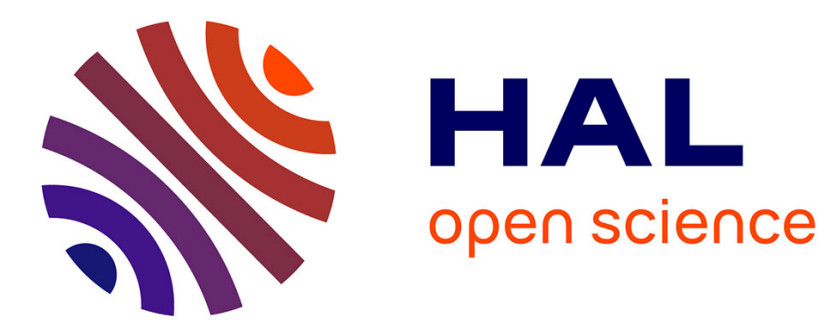

\title{
Diffusion Raman dans une fibre optique en verre fluoré
}

\author{
A. Saïssy, J. Botineau, L. Macon, G. Maze
}

\section{To cite this version:}

A. Saïssy, J. Botineau, L. Macon, G. Maze. Diffusion Raman dans une fibre optique en verre fluoré. Journal de Physique Lettres, 1985, 46 (6), pp.289-294. 10.1051/jphyslet:01985004606028900 . jpa00232513

\section{HAL Id: jpa-00232513 https://hal.science/jpa-00232513}

Submitted on 1 Jan 1985

HAL is a multi-disciplinary open access archive for the deposit and dissemination of scientific research documents, whether they are published or not. The documents may come from teaching and research institutions in France or abroad, or from public or private research centers.
L'archive ouverte pluridisciplinaire HAL, est destinée au dépôt et à la diffusion de documents scientifiques de niveau recherche, publiés ou non, émanant des établissements d'enseignement et de recherche français ou étrangers, des laboratoires publics ou privés. 
Classification

Physics Abstracts

$78.30-42.65 \mathrm{C}-42.80$

\title{
Diffusion Raman dans une fibre optique en verre fluoré
}

\author{
A. Saïssy, J. Botineau, L. Macon \\ Laboratoire d'Electro-Optique (*), Parc Valrose, 06034 Nice Cedex, France
}

et G. Maze

« Le Verre Fluoré S.A. », Z.I. du Champ Martin, Vern-sur-Seiche, 35230 Saint Erblon, France

(Reçu le 22 octobre 1984, accepté le 28 janvier 1985)

\begin{abstract}
Résumé. - Le spectre de la diffusion Raman spontanée d'un verre fluoré du système $\mathrm{ZrF}_{4}-\mathrm{BaF}_{2}$ est étudié au moyen d'une fibre optique. Une bande principale à $580 \mathrm{~cm}^{-1}$ et cinq bandes satellites moins intenses sont mises en évidence et interprétées à partir d'un assemblage de motifs élémentaires. Par la suite, nous comparons le rendement de la diffusion Raman stimulée dans les fibres en verre fluoré et en silice.

Abstract. - We study spontaneous Raman scattering in $\mathrm{ZrF}_{4}-\mathrm{BaF}_{2}$ fluorozirconate glass fiber. A main band at $580 \mathrm{~cm}^{-1}$ and five weaker bands are observed, each band have been assigned to motions in a structural unit assembly. We compare stimulated Raman scattering gain in fluorozirconate and silica fibers.
\end{abstract}

\section{Introduction.}

L'étude de la diffusion Raman dans les fibres optiques permet d'accéder aux propriétés structurelles des matériaux constituant la fibre [1], d'autre part, lorsque cette diffusion devient stimulée, elle conduit à la réalisation de sources lumineuses accordables [2]. Les propriétés spectrales (glissement de fréquence, largeur de raie, ...) de ce type de source dépendent étroitement de la nature du matériau constituant le coeur de la fibre; pratiquement ce dernier est constitué de silice, pure ou dopée, ou d'un liquide d'indice élevé [3].

Actuellement, il est possible de réaliser des fibres à partir de verres fluorés [4], aussi la réalisation d'une source lumineuse à diffusion Raman stimulée dans une fibre à verre fluoré est-elle envisageable. Les pertes optiques des verres fluorés sont théoriquement plus faibles et situées plus dans l'infrarouge moyen que pour la silice, aussi, la source lumineuse envisagée ci-dessus possédera de meilleures performances dans l'infrarouge moyen.

Par ailleurs l'étude du spectre de diffusion Raman spontanée d'une fibre constitue un moyen d'investigation non destructif pour connaître les propriétés structurelles du matériau à l'état présent dans la fibre. En effet la réalisation d'une fibre à partir d'une préforme passe par l'opération de fibrage qui a priori peut modifier la structure du matériau.

$\left(^{*}\right)$ LA 198 du CNRS. 
L'objet de la présente note est dans un premier temps l'étude des propriétés structurelles du verre fluoré conditionné sous forme de fibre optique, par la suite, nous étudions la mise en œuvre d'une diffusion Raman stimulée dans une fibre à verre fluoré.

\section{Conditions expérimentales.}

Les échantillons utilisés se présentent sous forme massive ou de fibres à cœur de silice ou de verre fluoré. Le verre fluoré est un fluorozirconate comportant approximativement $60 \% \mathrm{de} \mathrm{ZrF}_{4}, 30 \%$ de $\mathrm{BaF}_{2}$ (modificateur) et des stabilisateurs dans les proportions suivantes $3 \% \mathrm{LaF}_{3}, 4 \% \mathrm{AIF}_{3}$, $3 \% \mathrm{NaF}$. Le cour et la gaine optique de la fibre sont en verre fluoré avec des diamètres respectifs de $65 \mu \mathrm{m}$ et $155 \mu \mathrm{m}$, l'enrobage étant formé d'une résine U.V. avec un diamètre de $250 \mu \mathrm{m}$. Cette fibre est multimode, sans maintien de la polarisation lumineuse et de longueur $10 \mathrm{~m}$. Les spectres de diffusion Raman sont relevés à partir de la lumière sortant de la fibre, ils correspondent donc à une diffusion codirective sans sélection de polarisation. La source associée au spectromètre Raman est un laser à argon réglé sur $5145 \AA$ avec une puissance de quelques centaines de milliwatts que l'on couple à la fibre à l'aide d'un objectif de microscope $\times 20$. Le spectre de la lumière diffusée est relevé à l'aide d'un monochromateur à double réseau holographique Jobin \& Yvon, suivi d'un photomultiplicateur, d'une chaîne de photocomptage et d'un enregistreur.

\section{Résultats et discussion.}

3. 1 DifFUSION RAMAN SPONTANÉE. - Le spectre de diffusion Raman d'une fibre en verre fluoré dans le domaine spectral de 0 à $700 \mathrm{~cm}^{-1}$ est représenté sur la figure 1 simultanément avec le spectre d'une fibre en silice [5]. Ces spectres sont normalisés sur la bande de diffusion principale qui se situe à $580 \mathrm{~cm}^{-1}$ pour le verre fluoré et $450 \mathrm{~cm}^{-1}$ pour $\mathrm{SiO}_{2}$, soit un écart de $130 \mathrm{~cm}^{-1}$. La bande basse fréquence $\left(\sim 50 \mathrm{~cm}^{-1}\right)$ commune à tous les systèmes désordonnés est aussi présente dans le verre fluoré. L'intervalle spectral entre ces deux bandes est occupé par cinq bandes satellites situées à $480 \mathrm{~cm}^{-1}, 390 \mathrm{~cm}^{-1}, 330 \mathrm{~cm}^{-1}, 280 \mathrm{~cm}^{-1}$ et $205 \mathrm{~cm}^{-1}$ pour le verre fluoré, par contre, pour la silice il n'existe que deux bandes satellites, $490 \mathrm{~cm}^{-1}$ et $600 \mathrm{~cm}^{-1}$, situées au-delà de la bande principale. Les bandes principales ont des largeurs à mi-hauteur différentes dans ces deux verres, soit $100 \mathrm{~cm}^{-1}$ pour le verre fluoré et $300 \mathrm{~cm}^{-1}$ pour $\mathrm{SiO}_{2}$ d'où un écart de $200 \mathrm{~cm}^{-1}$; pour les bandes satellites, la situation est inversée.

Dans l'hypothèse d'un processus de diffusion du premier ordre [6], l'intensité lumineuse diffusée $I(\omega)$ par un matériau amorphe a pour expression :

$$
I(\omega)=\sum_{b} C_{b}[n(\omega, T)+1] g_{b}(\omega) / \omega
$$

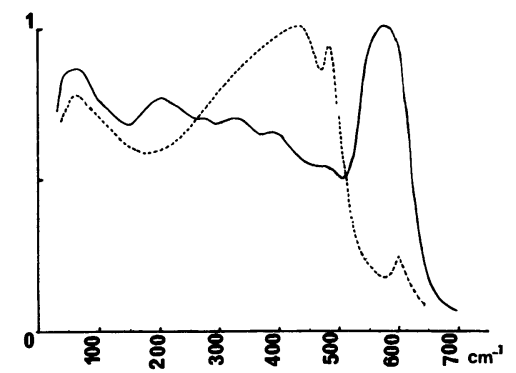

Fig. 1. - Spectres Raman (...) silice, (一) verre fluoré.

[Raman spectra (...) silice glass, (-) fluorozirconate glass.] 
où $n(\omega, T)$ est la distribution de Bose-Einstein à la température $T, \omega$ le déplacement de fréquence et $g_{b}(\omega)$ la densité d'états vibratoires de la bande $b$. Les propriétés structurelles du verre sont décrites par $g_{b}(\omega)$, aussi pour s'affranchir de la distribution thermique des phonons utiliseronsnous le spectre réduit :

$$
I_{\mathrm{r}}(\omega)=I(\omega) /[n(\omega, T)+1] .
$$

Les spectres réduits normalisés pour le verre fluoré et la silice sont portés sur la figure 2. L'importance de la bande $50 \mathrm{~cm}^{-1}$ est atténuée dans le spectre réduit, ce qui établit son lien avec la distribution thermique des phonons [7].

Pour affecter chaque bande à un état vibratoire particulier du système matériel, il faut supposer un modèle de structure vitreuse. A notre connaissance, il n'existe pas d'étude de diffusion Raman spontanée dans une fibre optique en verre fluoré, aussi, nous nous référerons à deux études : une de R. M. Almeida et J. D. Mackenzie [8] sur des échantillons massifs, l'autre de Y. Kawamoto [9] sur poudres pressées. La comparaison de nos spectres avec ceux correspondant à ces deux études nous montre que la position spectrale de la bande principale $580 \mathrm{~cm}^{-1}$ ainsi que sa largeur $100 \mathrm{~cm}^{-1}$ à sa base sur le front basse fréquence sont des constantes des différents spectres. Le spectre sur poudre ne présente pas de bandes satellites bien marquées, pour la fibre, nous relevons la présence d'une bande peu intense à $280 \mathrm{~cm}^{-1}$ non signalée par $\mathbf{R}$. M. Almeida, ceci résulte de l'augmentation du rendement de la diffusion dans une configuration de guide d'onde. Il résulte que le fibrage n'apporte pas de modification structurelle importante pour le verre fluoré constituant la fibre.

En ce qui concerne le modèle de structure vitreuse, $R$. M. Almeida propose l'existence de chaînes d'octaèdres $\mathrm{ZrF}_{6}$ liés par deux atomes de fluor pontant (Fig. 3). Par contre, pour Y. Kawamoto

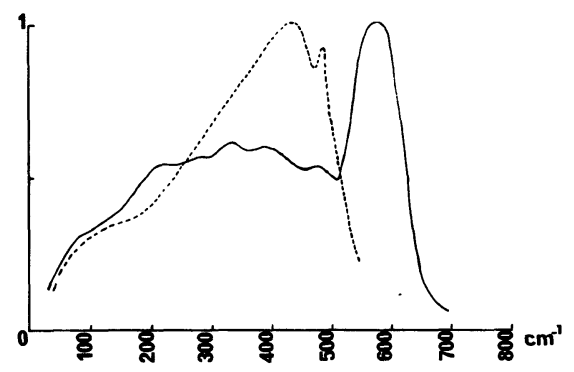

Fig. 2. - Spectres Raman réduits (...) silice vitreuse, (-) verre fluoré.

[Reduced Raman spectra (...) silice glass, (-) fluorozirconate glass.]

a)

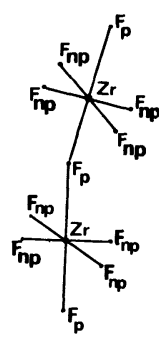

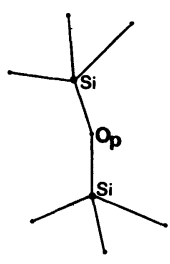

b)

Fig. 3. - Modèles de structure : (a) verre fluoré, (b) silice vitreuse.

[Basic structure : (a) fluorozirconate glass, (b) silica glass.] 
il s'agit plutôt de dodécaèdres $\mathrm{ZrF}_{8}$. La dernière hypothèse paraît la plus probable car les études de diffraction X [10] indiquent une coordinance 8 pour le zirconium. Dans les deux modèles nous retrouvons une structure analogue à celle proposée par S. Brawer pour les verres disilicates [11], une chaîne constituée de cellules élémentaires (tétraèdres $\mathrm{SiO}_{4}$, octaèdre $\mathrm{ZrF}_{6}$ ou dodécaèdre $\mathrm{ZrF}_{8}$ ) reliées par deux sommets (oxygène ou fluor pontant), les liaisons interchaînes étant réalisées par les atomes modificateurs (alcalins $\mathrm{Na}, \mathrm{Li}$ ou baryum). La bande principale $580 \mathrm{~cm}^{-1}$ est associée à la vibration d'élongation symétrique des liaisons zirconium-fluor non pontant dans la cellule élémentaire. La finesse de cette bande nous indique que le mouvement des atomes de fluor est peu amorti. La présence d'une bande principale intense avec une bande satellite proche moins intense est une situation que l'on rencontre aussi dans les verres disilicates, ceci suggère aussi l'analogie de structure de ces deux types de verre. Ainsi la bande $480 \mathrm{~cm}^{-1}$ peut être associée à un mouvement d'élongation anti-symétrique des atomes de fluor pontant. En ce qui concerne les autres bandes de diffusion, on peut se référer suivant R. M. Almeida soit à des mouvements internes aux cellules élémentaires (octaèdre ou dodécaèdre) soit à des mouvements d'ensemble de la chaîne de cellules élémentaires. Pour le rôle du baryum, les études de diffusion $\mathrm{X}$ ont montré qu'il existait deux types de distance $\mathrm{Ba}-\mathrm{F}$ de valeurs $0,26 \mathrm{~nm}$ et $0,30 \mathrm{~nm}$ [10], ce qui est plus grand que la valeur $0,21 \mathrm{~nm}$ pour $\mathrm{Zr}-\mathrm{F}$; les fréquences associées aux vibrations de la liaison $\mathrm{Ba}-\mathrm{F}$ seront donc plus basses. Ainsi le spectre Raman d'une fibre en verre fluoré (Fig. 2) concerne principalement la dynamique des liaisons $\mathrm{Zr}-\mathrm{F}$, l'influence des atomes de baryum ne se manifeste pas par l'apparition de bandes importantes. L'étude de la diffusion Raman spontanée dans une fibre nous a permis de vérifier que le pic principal de diffusion à $580 \mathrm{~cm}^{-1}$ conserve sa finesse lors du fibrage. Ce résultat est favorable pour la mise en œuvre de la diffusion Raman stimulée que nous envisagerons maintenant.

3.2 Diffusion Raman stimuléE. - De par leur faible section et leur longueur importante, les fibres optiques constituent des structures très favorables pour la stimulation de la diffusion Raman. Ainsi une onde lumineuse excitatrice de pulsation $\omega_{\mathrm{p}}$ et de puissance $P$ se propageant dans une fibre de longueur $l$ constituée d'un matériau actif en Raman à la pulsation $\omega_{\mathrm{v}}$ avec un gain $G\left(\omega_{\mathrm{v}}\right)$ va créer une onde lumineuse de pulsation $\omega_{\mathrm{s}}=\omega_{\mathrm{p}}-\omega_{\mathrm{v}}$ d'intensité $I_{\mathrm{s}} \sim \exp \frac{G P}{A_{\text {eff }}} l_{\text {eff }}$ avec $l_{\text {eff }}=\frac{1-\mathrm{e}^{-\alpha l}}{\alpha}$ longueur effective de la fibre et $A_{\text {eff }}$ sa section effective.

Le facteur de gain Raman a pour expression :

$$
G=\frac{16 \pi^{2} n_{\mathrm{p}}}{C n_{\mathrm{s}} \lambda_{\mathrm{s}}} \frac{f}{\Gamma\left[n\left(\omega_{\mathrm{v}}, r\right)+1\right]}
$$

où $f$ est la force d'oscillateur de la vibration, $f /[n(\omega, \Upsilon)+1]$ est proportionnel à l'intensité réduite diffusée, $2 \Gamma$ est la largeur à mi-hauteur de cette courbe. Ainsi sur la figure 2 on constate que la bande de diffusion principale à $580 \mathrm{~cm}^{-1}$ du verre fluoré avec une largeur à mi-hauteur de $100 \mathrm{~cm}^{-1}$ devrait avoir un gain Raman supérieur à celui de la silice à $450 \mathrm{~cm}^{-1}$ où la largeur de bande est $300 \mathrm{~cm}^{-1}$. Pour vérifier cette hypothèse nous avons réalisé une expérience de diffusion Raman stimulée. Un laser YAG à fréquence doublée délivrant des impulsions lumineuses de durée $10 \mathrm{~ns}$ à la longueur d'onde $5320 \AA$ est couplé dans la fibre en verre fluoré décrite ci-dessus. La lumière sortant de la fibre est analysée avec un monochromateur à réseau de faible résolution. On observe une raie Stokes peu intense à la longueur d'onde $5490 \AA, \omega_{v}=582 \mathrm{~cm}^{-1}$, pour des puissances lumineuses excitatrices de quelques kilowatts voisines du seuil de rupture de la face d'entrée de la fibre. D'autre part, l'intensité lumineuse en champ lointain à la sortie de la fibre présente un anneau rouge pour les impulsions lumineuses les plus intenses.

Pour des puissances lumineuses du même ordre de grandeur, nous avons obtenu plusieurs raies Stokes dans des fibres en silice de faible perte [12]. Pour comparer avec le résultat ci-dessus, 
considérons le cas d'une fibre de $500 \mathrm{db} / \mathrm{km}$ de perte à $0,53 \mu \mathrm{m}$, de longueur $10 \mathrm{~m}$ et de diamètre de cour $65 \mu \mathrm{m}$, sa longueur effective sera $5,9 \mathrm{~m}$. Une fibre en silice de facteur de gain Raman $G=2 \times 10^{-11} \mathrm{~cm} / \mathrm{W}$, d'ouverture numérique telle que sa surface effective soit égale à la moitié de la surface géométrique donnera une amplification de $10^{3}$ pour une puissance excitatrice de $10 \mathrm{~kW}$, dans ce cas on n'observera pas de raies Stokes. Pour une fibre en verre fluoré, le facteur de gain Raman sera 3 fois plus grand en supposant la force d'oscillateur $f$ constante, elle donnera une amplification de $10^{9}$ ce qui est suffisant pour observer une faible raie Stokes.

Pour comparer les forces d'oscillateurs $f$ de la silice et du verre fluoré, nous avons mesuré dans des conditions expérimentales comparables, le rapport des intensités du pic principal de diffusion du verre fluoré et de la silice, nous trouvons $1 \pm 0,25$. Ce résultat ne remet pas en question l'augmentation de gain relative à la diminution de la largeur de la bande de diffusion.

Les faits expérimentaux et la comparaison ci-dessus sont donc en accord, ce qui confirme la supériorité du facteur de gain Raman du verre fluoré sur la silice. Pour obtenir un meilleur rendement Raman, il est souhaitable de diminuer les pertes de la fibre [13], une diminution des pertes est prévue théoriquement [14] sa réalisation pratique est à l'étude. Une deuxième voie est la réduction de la section de la fibre de manière à augmenter l'intensité lumineuse, un choix convenable de la section et de la différence d'indice cœur-gaine permet en outre d'éliminer ou de renforcer les effets non linéaires annexes du type mélange à quatre ondes [15]. Une troisième voie à explorer serait la réalisation de fibres en verre fluoré à maintien de polarisation.

Comme nous pouvons le constater, il existe encore de nombreuses voies à explorer pour améliorer le rendement de la diffusion Raman stimulée dans les fibres en verre fluoré. Néanmoins, les résultats préliminaires présentés ci-dessus nous montrent qu'il est maintenant possible de disposer de fibres en verre fluoré capables de donner lieu à de la diffusion Raman stimulée.

\section{Conclusion.}

Nous avons étudié la diffusion Raman spontanée dans une fibre en verre fluoré. La comparaison du spectre de cette diffusion avec celui obtenu sur des verres massifs nous a permis de vérifier que le fibrage n'apporte pas de modification structurelle du verre. D'autre part, la comparaison avec le spectre d'une fibre en silice fait apparaître un glissement de la bande principale de $450 \mathrm{~cm}^{-1}$ pour $\mathrm{SiO}_{2}$ à $580 \mathrm{~cm}^{-1}$ pour le verre fluoré ainsi qu'une réduction de $200 \mathrm{~cm}^{-1}$ de la largeur de la bande du $\mathrm{SiO}_{2}$. Cette dernière se manifeste par une augmentation du gain Raman en diffusion stimulée que nous avons mis en évidence expérimentalement avec un faible rendement, des solutions sont proposées pour améliorer cette situation.

Notre étude se situe dans le visible, ce qui est justifié tant que la diminution des pertes optiques dans l'infrarouge moyen ne compense pas la diminution du gain Raman lié à l'augmentation de la longueur d'onde. Un abaissement, prévisible des pertes optiques devrait permettre l'extension ainsi que l'amélioration des résultats ci-dessus dans l'infrarouge moyen.

Ainsi, les propriétés structurelles particulières du verre fluoré confèrent aux fibres optiques des possibilités nouvelles dans la réalisation d'une source lumineuse à diffusion Raman stimulée dans une fibre.

\section{Remerciements.}

Nous remercions F. Fried du LA 190 pour son aimable collaboration à cette étude et J. Lucas de Rennes pour les échantillons massifs. 


\section{Bibliographie}

[1] Walrafen, G. E., Stone, J., Appl. Spectrosc. 29 (1975) 337.

[2] Stolen, R. H., Ippen, E. P., Tynes, A. R., Appl. Phys. Lett. 20 (1972) 62.

[3] Chraplyvy, A. R., Bridges, T. J., Opt. Lett. 6 (1981) 632.

[4] Maze, G., Cardin, V., Poulain, M., Proc. Soc. Photo-Opt. Instrum. Eng. 400 (1983) 60.

[5] Saïssy, A., Botineau, J., Azema, A., Gires, F., J. Phys. Lett. 40 (1979) 355.

[6] Shuker, R., Gamnon, R. W., Phys. Rev. Lett. 25 (1970) 222.

[7] Hass, M., J. Phys. Chem. Solids 31 (1970) 415.

[8] Almeida, R. M., Mackenzie, J. D., J. Chem. Phys. 74 (1981) 5954.

[9] Kawamoto, Y., Physics and Chemistry of Glasses, Vol. 25, $\mathrm{n}^{\circ}$ 3, June 1984.

[10] Coupe, R., Louer, D., Lucas, J., Leonard, A. J., J. Am. Ceram. Soc. 66 (1983) 523.

[11] Brawer, S., Phys. Rev. B 11 (1975) 3173.

[12] Saïssy, A., Azema, A., Botineau, J., Gires, F., J. Phys. Lett. 40 (1979) 23.

[13] Maze, G., Cardin, V., Poulain, M., IEEE J. Lightwave Technology, vol. LT 2 (1984) 596.

[14] Shibata, S., Horiguchi, M., Jinguchi, K., Mitachi, S., Kanamori, K., Manabe, T., Electron. Lett. 17 (1981) 775.

[15] Saissy, A., Botineau, J., Azema, A., Gires, F., Appl. Opt. 19 (1980) 1639.

[16] Stolen, R. H., IEEE J. Quantum Electron. QE 15 (1979) 1157. 